\title{
ASYMPTOTIC EQUIVALENCE AND ASYMPTOTIC BEHAVIOUR OF LINEAR SYSTEMS
}

\author{
Fred Brauer
}

1. Two systems of differential equations are said to be asymptotically equivalent if, corresponding to each solution of one system, there exists a solution of the other system such that the difference between these two solutions tends to zero. If we know that two systems are asymptotically equivalent, and if we also know the asymptotic behaviour of the solutions of one of the systems, then it is clear that we can obtain information about the asymptotic behaviour of the solutions of the other system. In some cases, we can reduce the problem of asymptotic equivalence to the problem of proving that a certain system has solutions which tend to any prescribed limit. Then, by using slight extensions of some results of Wintner [10], we obtain alternate proofs of some theorems of Wintner [8] on asymptotic equivalence, as well as some results analogous to those of Levinson [6] and Yakubovič [12] on asymptotic behaviour of linear perturbations of linear systems with constant coefficients. In general, our hypotheses are considerably more stringent than Levinson's, but our error estimates are correspondingly sharper. The same approach yields results analogous to those of Cesari [2] and Levinson [6] on asymptotic behaviour of certain linear systems. Here too, our hypotheses are more stringent, but our error estimates are sharper than earlier results. Unfortunately, we see no way to obtain the earlier results from ours. Our proofs are simpler than previous proofs, in that they avoid conversion to integral equations and construction of successive approximations. Instead, they depend on the results of Wintner [10] mentioned earlier, whose proofs are quite elementary.

We shall always write systems of differential equations in the usual vector form (see for example [3] or [4]). The norm $|x|$ of a vector $x$ will always mean the sum of the absolute values of the components of $x$, and the norm $|A|$ of a matrix $A$ will always mean the sum of the absolute values of the elements of A. All derivatives with respect to $t$ will be denoted by ', and the derivative of a vector or matrix will always mean the vector or matrix respectively whose elements are the derivatives of the elements of the original vector or matrix. We shall assume that all coefficients are continuous, without always stating this explicitly.

2. We wish to compare the solutions of the linear system

$$
x^{1}=A(t) x
$$

with the solutions of a perturbed system

$$
\mathrm{y}^{\prime}=\mathrm{A}(\mathrm{t}) \mathrm{y}+\mathrm{f}(\mathrm{t}, \mathrm{y}) .
$$

Sometimes we shall be interested in a linear perturbation giving a system

$$
y^{\prime}=[A(t)+B(t)] y .
$$

Received September 28, 1961.

This research was supported by the National Science Foundation, Grant G 10093. 
Here, $\mathrm{x}, \mathrm{y}$, and $\mathrm{f}$ are $\mathrm{n}$-dimensional column vectors, and $\mathrm{A}$ and $\mathrm{B}$ are $\mathrm{n} \times \mathrm{n}$ matrices.

The systems (1) and (2) are said to be asymptotically equivalent if to each solution $x(t)$ of (1) there corresponds a solution $y(t)$ of (2) such that

$$
\lim _{t \rightarrow \infty}[y(t)-x(t)]=0 \text {, }
$$

and if, conversely, to each solution $y(t)$ of (2) there corresponds a solution $x(t)$ of (1) such that (4) holds. Let $X(t)$ be a fundamental matrix of (1), and set

$y(t)=X(t) v(t)$. Then it is easy to verify that $y(t)$ is a solution of (2) if and only if $v(t)$ satisfies

$$
v^{\prime}=X^{-1}(t) f(t, X(t) v)
$$

The verification is simply the method of variation of constants. Any solution $x(t)$ of (1) can be written $x(t)=X(t) c$, where $c$ is a constant column vector. Suppose that all solutions of (5) tend to limits as $t \rightarrow \infty$, and that there exists a solution $v(t)$ of (5) such that $\lim _{t \rightarrow \infty} v(t)=c$. This $v(t)$ gives a solution $y(t)$ of $(2)$ such that

$$
y(t)-x(t)=x(t)[v(t)-c]
$$

If it is known that all solutions of $(1)$ are bounded, so that $X(t)$ is bounded, this implies (4), and the asymptotic equivalence of (1) and (2) follows. We state this result as a theorem, essentially a restatement of a theorem of Wintner [8], who treated the linear case.

THEOREM 1. Suppose that all solutions of (1) are bounded as $\mathrm{t} \rightarrow \infty$. Suppose also that every solution of (5) tends to a finite limit vector as $t \rightarrow \infty$, and that there exists a solution of (5) which tends to any prescribed vector as $t \rightarrow \infty$. Then (1) and (2) are asymptotically equivalent.

To apply this theorem in any particular case, we need a result such as the following one, due to Wintner $[7,10]$, whose proof may also be found in $[3$, p. 43].

LEMMA 1. If $\int^{\infty}|\mathrm{D}(\mathrm{t})| \mathrm{dt}<\infty$, then every solution $\mathrm{v}(\mathrm{t})$ of

$$
\mathrm{v}^{\prime}=\mathrm{D}(\mathrm{t}) \mathrm{v}
$$

tends to a finite limit vector as $\mathrm{t} \rightarrow \infty$, and, given any finite vector, there exists a solution of (6) which tends to this vector as $\mathrm{t} \rightarrow \infty$.

We shall generalize this lemma slightly in the next section, still restricting it to linear systems. Actually, Wintner proved the following result for non-linear systems in $[9,10]$.

LEMMA 2. Let $\lambda(\mathrm{t})$ be a continuous non-negative function on $0 \leq \mathrm{t}<\infty$, with
$\int_{0}^{\infty} \lambda(\mathrm{t}) \mathrm{dt}<\infty$. If the system $\mathrm{v}^{\prime}=\mathrm{g}(\mathrm{t}, \mathrm{v})$ satisfies the condition

$$
|\mathrm{g}(\mathrm{t}, \mathrm{v})| \leq|\mathrm{v}| \lambda(\mathrm{t}) \quad(0 \leq \mathrm{t}<\infty,|\mathrm{v}|<\infty)
$$

then each of its solutions tends to a finite limit vector as $t \rightarrow \infty$, and each finite vector is the limit (as $t \rightarrow \infty$ ) of one of its solutions. 
For a linear perturbed equation of the form (3), the equation (5) takes the form

$$
v^{\prime}=X^{-1}(t) B(t) X(t) v
$$

If $X(t)$ and $X^{-1}(t)$ are bounded and $\int^{\infty}|B(t)| d t<\infty$, Lemma 1 gives the asymptotic equivalence of (1) and (3). The boundedness of $x^{-1}(t)$ is assured by the hypothesis

$$
\liminf _{t \rightarrow \infty} \int_{0}^{t} \operatorname{tr} A(s) d s>-\infty
$$

and this gives another result of Wintner [8].

THEOREM 2. Suppose that all solutions of (1) are bounded as $t \rightarrow \infty$, that (8) is satisfied, and that $\int^{\infty}|\mathrm{B}(\mathrm{t})| \mathrm{dt}<\infty$. Then the linear systems (1) and (3) are asymptotically equivalent.

Using Lemma 2, we can treat non-linear perturbations dominated by linear terms as in (2). It is easy to obtain the following slight extension of Theorem 2.

THEOREM 3. Suppose that all solutions of (1) are bounded as $\mathrm{t} \rightarrow \infty$, that (8) is satisfied, and that $|\mathrm{f}(\mathrm{t}, \mathrm{y})| \leq|\mathrm{y}| \lambda(\mathrm{t})$, where $\int^{\infty} \lambda(\mathrm{t}) \mathrm{dt}<\infty$. Then the systems (1) and (2) are asymptotically equivalent.

The proof follows from the inequality

$$
\left|X^{-1}(t) f(t, X(t) v)\right| \leq\left|X^{-1}(t)\right||X(t)||v| \lambda(t) \leq K|v| \lambda(t)
$$

valid for some constant $\mathrm{K}$, and Lemma 2 and Theorem 1.

3. Now we shall discuss the asymptotic behaviour of solutions of linear systems. First we require some extensions of Lemma 1.

LEMMA 3. If $\mathrm{v}(\mathrm{t})$ is a solution of the linear system (6) with $\lim _{\mathrm{t} \rightarrow \infty} \mathrm{v}(\mathrm{t})=\mathrm{c}$, and if $|\mathrm{D}(\mathrm{t})| \leq \lambda(\mathrm{t})$, where $\int^{\infty} \lambda(\mathrm{t}) \mathrm{dt}<\infty$, then there exists a constant $\mathrm{K}$ such that $|\mathrm{v}(\mathrm{t})-\mathrm{c}| \leq \mathrm{K} \int_{\mathrm{t}}^{\infty} \lambda(\mathrm{u}) \mathrm{du}$.

Proof.

$$
|v(t)-c|=\left|\int_{t}^{\infty} D(u) v(u) d u\right| \leq \int_{t}^{\infty}|D(u)||v(u)| d u \leq \int_{t}^{\infty} \lambda(u)|v(u)| d u
$$

It has been shown by Wintner [11, Appendix] (see also [1], [5]) that if $r(t)$ is the solution of $r^{\prime}=\lambda(t) r$ with $r(0)=|v(0)|$, then $|v(t)| \leq r(t)$ for $t \geq 0$. Clearly, $r(t)$ is monotone increasing and tends to a finite limit $r_{\infty}$ as $t \rightarrow \infty$. This implies that

$$
|v(t)-c| \leq \int_{t}^{\infty} \lambda(u) r(u) d u=\int_{t}^{\infty} r^{\prime}(u) d u=r_{\infty}-r(t)
$$

To estimate $r_{\infty}-r(t)$, we observe that each solution $r(t)$ of $r^{\prime}=\lambda(t) r$ has the form 


$$
r(t)=a e^{\int_{0}^{t} \lambda(u) d u}
$$

where $a=r(0)=|v(0)|$, and that it therefore tends to the limit

$$
r_{\infty}=a e^{\int_{0}^{\infty} \lambda(u) d u}
$$

Consequently,

$$
r_{\infty}, r(t)=a e^{\int_{0}^{\infty} \lambda(u) d u}\left(1-e^{-\int_{t}^{\infty} \lambda(u) d u}\right) \leq a e^{\int_{0}^{\infty} \lambda(u) d u} \int_{t}^{\infty} \lambda(u) d u,
$$

by the elementary inequality $1-e^{-b} \leq b$ for $b \geq 0$. The result now follows, with the constant

$$
K=|v(0)| e^{\int_{0}^{\infty} \lambda(u) d u}
$$

depending only on the initial value $|\mathrm{v}(0)|$.

LEMMA 4. For the non-homogeneous system

$$
v^{\prime}=D(t) v+a(t)
$$

with $|\mathrm{D}(\mathrm{t})| \leq \lambda(\mathrm{t}),|\mathrm{a}(\mathrm{t})| \leq \lambda(\mathrm{t})$, and $\int^{\infty} \lambda(\mathrm{t}) \mathrm{dt}<\infty$, every solution tends to a finite limit vector as $\mathrm{t} \rightarrow \infty$, and to each finite vector there corresponds a solution of (9) which tends to this vector as $\mathrm{t} \rightarrow \infty$. If $\mathrm{v}(\mathrm{t})$ is a solution of $(9)$ with $\lim _{\mathrm{t} \rightarrow \infty} \mathrm{v}(\mathrm{t})=\mathrm{c}$, then

$$
|v(t)-c| \leq K \int_{t}^{\infty} \lambda(u) d u
$$

for some constant $\mathrm{K}$.

Proof. As in the proof of Lemma 3, we obtain

$$
|v(t)-c| \leq \int_{t}^{\infty}[\lambda(u)|v(u)|+\lambda(u)] d u
$$

If $r(t)$ is the solution of $r^{\prime}=\lambda(t) r+\lambda(t)$ with $r(0)=|v(0)|$, then $|v(t)| \leq r(t)$ for $t \geq 0$, as in Lemma 3, and $r(t)$ is monotone increasing and tends to a limit $r_{\infty}$ as $t \rightarrow \infty$. Again $|v(t)-c| \leq r_{\infty}-r(t)$, and we must estimate $r_{\infty}-r(t)$. Any nontrivial solution $r_{0}(t)$ of the corresponding homogeneous equation $r^{\prime}=\lambda(t) r$ tends to a limit $r_{0}(\infty)$ as $t \rightarrow \infty$, and $r_{0}(\infty)-r_{0}(t) \leq K \int_{t}^{\infty} \lambda(u) d u$, by Lemma 3 . We can write

$$
r(t)=r_{0}(t)-r_{0}(t) \int_{t}^{\infty}\left[\lambda(u) / r_{0}(u)\right] d u
$$


for a suitable homogeneous solution $r_{0}(t)$, and $r_{\infty}=r_{0}(\infty)$. Then

$$
r_{\infty}-r(t)=r_{0}(\infty)-r_{0}(t)+r_{0}(t) \int_{t}^{\infty}\left[\lambda(u) / r_{0}(u)\right] d u
$$

Since $r_{0}(t)$ is monotone increasing, $r_{0}(t) / r_{0}(u) \leq 1$ for $t \leq u$, and

$$
r_{0}(t) \int_{t}^{\infty}\left[\lambda(u) / r_{0}(u)\right] d u \leq \int_{t}^{\infty} \lambda(u) d u
$$

The bound on $r_{0}(\infty)-r_{0}(t)$ given by Lemma 3 now yields the result.

We can now prove the main result of this section.

THEOREM 4. Let A in (1) be a constant matrix. Let the distinct real parts of the characteristic roots of $\mathrm{A}$ be $\mu_{\mathrm{i}}(\mathrm{i}=1, \cdots, \mathrm{s})$, with $\mu_{1}<\mu_{2}<\cdots<\mu_{\mathrm{s}}$. Let $\mathrm{m}_{\mathrm{i}}$ be the largest multiplicity of any characteristic root with real part $\mu_{\mathrm{i}}(\mathrm{i}=1, \cdots, \mathrm{s})$, and let $\mathrm{m}=\max _{1 \leq \mathrm{i} \leq \mathrm{s}} \mathrm{m}_{\mathrm{i}}$. Suppose

$$
\int^{\infty} \mathrm{t}^{\mathrm{a}} \mathrm{e}^{\left(\mu_{\mathrm{s}}-\mu_{1}+\mathrm{k}\right) \mathrm{t}}|\mathrm{B}(\mathrm{t})| \mathrm{dt}<\infty
$$

for some $\mathrm{k} \geq 0$ and some real a, subject to the restriction $\mathrm{a} \geq \mathrm{m}_{1}+\mathrm{m}_{\mathrm{s}}-2$ if $\mathrm{k}=0$. Then to each solution $\mathrm{x}(\mathrm{t})$ of $(1)$, there corresponds a solution $\mathrm{y}(\mathrm{t})$ of (3) such that

$$
y(t)-x(t)=o\left[t^{2 m+m_{s}-3-a} e^{\left(\mu_{1}-k\right) t}\right] \quad(t \rightarrow \infty) .
$$

Conversely, to each solution $\mathrm{y}(\mathrm{t})$ of $(3)$ there corresponds a solution $\mathrm{x}(\mathrm{t})$ of $(1)$ such that (11) holds.

Proof. There exists a constant matrix which transforms (3) to a system of the same form with the same integrability condition (10), but with A replaced by a suitable canonical form. Thus we may assume that $A$ has the form

$$
\mathrm{A}=\left(\begin{array}{ccccc}
\mathrm{A}_{1} & & & & \\
& \mathrm{~A}_{2} & & \\
& & \cdot & & \\
& & \cdot & \\
& & & \cdot & \\
& & & \mathrm{A}_{\mathrm{S}}
\end{array}\right)
$$

Each block $A_{i}$ corresponds to the characteristic roots with real part $\mu_{i}$. Then we can write

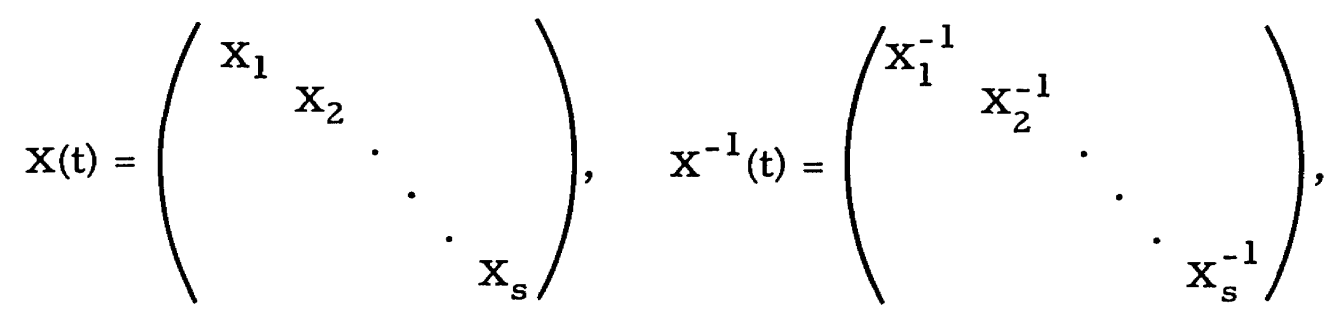

where 


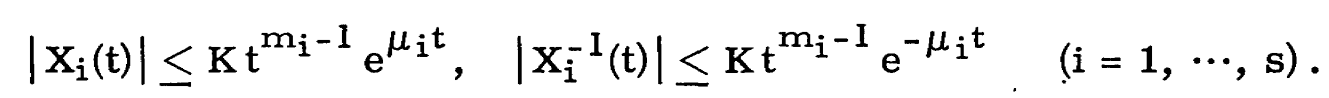

We can decompose the matrix $B(t)$ into blocks of the same size as the $A_{i}$. Thus, if $A_{i}$ is an $n_{i} \times n_{i}$ matrix, we obtain blocks $B_{i j}(t)$ with $n_{i}$ rows and $n_{j}$ columns $(i, j=1, \cdots, s)$, and

$$
B(t)=\left(\begin{array}{ccc}
B_{11}(t) & \cdots & B_{1 s}(t) \\
\cdots & \cdots & \cdots \\
B_{s 1}(t) & \cdots & B_{s s}(t)
\end{array}\right)
$$

The system (7) becomes

$$
v_{i}^{\prime}=\sum_{j=1}^{s} x_{i}^{-1}(t) B_{i j}(t) x_{j}(t) v_{j} \quad(i=1, \cdots, s),
$$

where $v$ is a vector decomposed into $s$ vectors $v_{i}$ of dimension $n_{i}$. In view of (12),

$$
\left|x_{i}^{-1}(t) B_{i j}(t) X_{j}(t)\right| \leq K t^{m_{i}+m_{j}-2} e^{\left(\mu_{j}-\mu_{i}\right) t}\left|B_{i j}(t)\right|
$$

Because of the assumption (10), each vector $c$ is the limit of a solution $v(t)$ of (13). Each equation in (13) (actually an $n_{i}$-dimensional system) can be regarded as a linear non-homogeneous system for $v_{i}$ with the non-homogeneous part consisting of all terms in $v_{j}$ for $j \neq i$. Then, because of (10) and (12), the equations in (13) can be written as $v_{i}^{i}=D_{i}(t) v_{i}+a_{i}(t)$, with $\left|D_{i}(t)\right| \leq \lambda_{i}(t),\left|a_{i}(t)\right| \leq \lambda_{i}(t)$, for

$$
\lambda_{i}(t)=K t^{m_{i}+m_{s}-2} e^{\left(\mu_{s}-\mu_{i}\right) t}\left|B_{i s}(t)\right|=t^{m_{i}+m_{s}-a-2} e^{-\left(\mu_{i}-\mu_{1}+k\right) t} q_{i}(t),
$$

for some non-negative integrable function $q_{i}(t)$. Then $n_{i}$-dimensional vector $v_{i}(t)$ tends to an $n_{i}$-dimensional vector $c_{i}$, made up of the appropriate components of $c$, and by Lemma 4 ,

$$
\left|v_{i}(t)-c_{i}\right| \leq K \int_{t}^{\infty} \lambda_{i}(u) d u \leq K \int_{t}^{\infty} u^{m_{i}+m_{s}-a-2} e^{-\left(\mu_{i}-\mu_{1}+k\right) u} q_{i}(u) d u
$$

Since $u^{m_{i}+m_{s}-a-2} e^{-\left(\mu_{i}-\mu_{1}+k\right) u}$ is monotone decreasing for sufficiently large $u$, we have

$$
\left|v_{i}(t)-c_{i}\right|=o\left[t^{m_{i}+m_{s}-a-2} e^{-\left(\mu_{i}-\mu_{1}+k\right) t}\right] \quad(t \rightarrow \infty) .
$$

Let $x_{i}(t)$ and $y_{i}(t)$ be the $n_{i}$-dimensional vectors whose components are the components of $x(t)$ and $y(t)$ corresponding to the components of $v(t)$ which appear in $v_{i}(t)$ for any solutions $x(t)$ of $(1)$ and $y(t)$ of (3). Then, corresponding to each solution $x(t)$ of $(1)$, with $x(t)=X(t) c$, there exists a solution $y(t)$ of $(3)$ with $y(t)=X(t) v(t)$. For these solutions, $x_{i}(t)=x_{i}(t) c_{i}$ and $y_{i}(t)=x_{i}(t) v_{i}(t)$. In view of (12) and (14),

$$
\left|y_{i}(t)-x_{i}(t)\right|=o\left(t^{2 m_{i}+m_{s}-a-3} e^{\left(\mu_{1}-k\right) t}\right)=o\left(t^{2 m+m_{s}-a-3} e^{\left(\mu_{1}-k\right) t}\right) \quad(t \rightarrow \infty) .
$$

Since this estimate holds for all $i$, we obtain (11). 
We note that in order to draw a conclusion $y(t)-x(t) \rightarrow 0$ as $t \rightarrow \infty$, we may take $\mathrm{k}=\mu_{1}$ and $\mathrm{a}=2 \mathrm{~m}+\mathrm{m}_{\mathrm{s}}-3$ if $\mu_{1} \geq 0$. Thus the condition

$$
\int^{\infty} t^{2 m+m_{s}-3} e^{\mu s}|B(t)| d t<\infty
$$

implies $\mathrm{y}(\mathrm{t})-\mathrm{x}(\mathrm{t}) \rightarrow 0$ as $\mathrm{t} \rightarrow \infty$. If $\mu_{1}<0$, we may take $\mathrm{a}=2 \mathrm{~m}+\mathrm{m}_{\mathrm{s}}-3$ and $\mathrm{k}=0$, and we see that

$$
\int^{\infty} t^{2 m+m_{s}-3} e^{\left(\mu_{s}-\mu_{1}\right) t}|B(t)| d t<\infty
$$

implies $y(t)-x(t)=o\left(e^{\mu_{1} t}\right)$.

Of course, Theorem 4 also applies to a non-linear perturbation (2) with $|f(t, y)| \leq|y| \lambda(t)$, where $\lambda(t)$ is a continuous non-negative scalar function satisfying the same integrability condition as $|B(t)|$. The proof is a straightforward adaptation of the proof of Theorem 3.

To indicate the relation between Theorem 4 and earlier results of this type, we quote two of these results.

THEOREM 5 (Levinson [6]). Let A be a constant matrix, and suppose the distinct real parts of the characteristic roots of $\mathrm{A}$ are $\mu_{\mathrm{i}}(\mathrm{i}=1, \cdots, \mathrm{s})$, with

$$
\mu_{1}<\mu_{2}<\cdots \mu_{\mathrm{m}}<-\beta<0 \leq \mu_{\mathrm{m}+1}<\cdots<\mu_{\mathrm{s}} .
$$

Suppose $\mathrm{B}(\mathrm{t})$ is a matrix with $\int^{\infty}|\mathrm{B}(\mathrm{t})| \mathrm{dt}<\infty$. Then there exist $\mathrm{n}$ linearly independent vector solutions $\mathrm{y}^{(\mathrm{k})}(\mathrm{t})(\mathrm{k}=1, \cdots, \mathrm{n})$ such that, as $\mathrm{t} \rightarrow \infty$,

$$
e^{\beta t} y^{(k)}(t) \rightarrow 0 \quad(k=1, \cdots, m), \quad y^{(k)}(t) \sim C^{(k)} e^{\mu} k^{t} \quad(k=m+1, \cdots, n),
$$

where the $\mathrm{C}^{(\mathrm{k})}$ are $\mathrm{n}-\mathrm{m}$ independent constant column vectors, characteristic vectors of the matrix $\mathrm{A}(\mathrm{k}=\mathrm{m}+1, \cdots, \mathrm{n})$.

THEOREM 6 (Yakubovic [12]). Let A be a constant matrix. Use the notation of Theorem 4 and, in addition, let $\mathrm{p}$ be the largest multiplicity of any characteristic root with real part zero, with the convention that $\mathrm{p}=1$ if there are no such characteristic roots. Consider a non-linear system (2) with $|\mathrm{f}(\mathrm{t}, \mathrm{y})| \leq|\mathrm{y}| \lambda(\mathrm{t})$. If

$$
\int^{\infty} t^{m_{s}+p-2} e^{\mu_{s} t} \lambda(t) d t<\infty
$$

then (1) and (2) are asymptotically equivalent.

Theorem 4 is weaker than Theorem 5 in the sense that it requires a much stronger integrability condition. However, it is stronger in the sense that it gives a sharper conclusion than the asymptotic relation (15).

If in Theorem 4 we take $a=m_{s}+p-2$ and $k=\mu_{1}$ when $\mu_{1} \geq 0$, we have the same hypotheses as in Theorem 6 . However, Theorem 4 yields only

$$
y(t)-x(t)=o\left(t^{2 m-p-1}\right),
$$

while Theorem 6 yields $y(t)-x(t)=o(1)$. If $m=1$, which implies $p=1$, the results are the same, but otherwise our result is weaker than Yakubovič's. However, under 
stronger integrability conditions, our result yields sharper estimates. In general, the smaller the perturbation, the smaller the difference between the solutions of the perturbed and unperturbed equations.

4. This section deals with linear perturbations of linear systems with variable coefficients. Let $\mathrm{A}$ be a constant matrix with distinct characteristic roots $\sigma_{1}, \cdots, \sigma_{n}$, with $\Re \sigma_{i}=\mu_{i}(i=1, \cdots, n)$ and $\mu_{1} \leq \mu_{2} \leq \cdots \leq \mu_{n}$. Let $V(t)$ be a differentiable matrix, and suppose $A+V(t)$ has characteristic $\operatorname{roots} \lambda_{1}(t), \cdots, \lambda_{n}(t)$, arranged in order of increasing real parts. Suppose $\lim _{t \rightarrow \infty} V(t)=0$. Then, by reordering the $\sigma_{i}$ if necessary, we can assume that $\lim \lambda_{i}(t)=\sigma_{i}(i=1, \cdots, n)$.

We now require an extension of a method of Cesari [2] for diagonalizing variable matrices.

LEMMA 6. Suppose that $\lim _{\mathrm{t} \rightarrow \infty} \mathrm{V}(\mathrm{t})=0$ and that $\mathrm{V}^{\prime}(\mathrm{t})$ satisfies some integrability condition of the form

$$
\int^{\infty} f(t)\left|V^{\prime}(t)\right| d t<\infty
$$

where $\mathrm{f}(\mathrm{t})$ is a non-negative continuous function. Then there exists a differentiable matrix $\mathrm{S}(\mathrm{t})$, which tends to a non-singular constant matrix $\mathrm{T}$ as $\mathrm{t} \rightarrow \infty$, such that $\mathrm{S}^{-1}(\mathrm{t})[\mathrm{A}+\mathrm{V}(\mathrm{t})] \mathrm{S}(\mathrm{t})=\Lambda(\mathrm{t})$, where $\Lambda(\mathrm{t})$ is a diagonal matrix whose diagonal elements are $\lambda_{1}(\mathrm{t}), \cdots, \lambda_{\mathrm{n}}(\mathrm{t})$. $\mathrm{S}(\mathrm{t})$ and $\mathrm{S}^{-1}(\mathrm{t})$ are bounded, and

$$
\int^{\infty} f(t)\left|S^{\prime}(t)\right| d t<\infty
$$

Proof. There exists a non-singular constant matrix $\mathrm{T}$ such that $\mathrm{T}^{-1} \mathrm{AT}=\hat{\mathrm{A}}$, where $\hat{A}$ denotes the diagonal matrix with diagonal elements $\sigma_{1}, \cdots, \sigma_{n}$. We let $\mathrm{S}=\mathrm{T} \hat{S}$, and we attempt to find $\hat{S}$. We require

$$
S^{-1}(A+V) S=\hat{S}^{-1} T^{-1}(A+V) T \hat{S}=\hat{S}^{-1}\left(T^{-1} A T+T^{-1} V T\right) \hat{S}=\hat{S}^{-1}(\hat{A}+\hat{V}) \hat{S},
$$

where $\hat{\mathrm{V}}=\mathrm{T}^{-1} \mathrm{VT}$. Since $\hat{\mathrm{V}}$ is linear and homogeneous in the elements of $\mathrm{V}$, it is differentiable and satisfies

$$
\lim _{t \rightarrow \infty} \hat{V}(t)=0, \quad \int^{\infty} f(t)\left|\hat{V}^{\prime}(t)\right| d t<\infty .
$$

Consider the matrix $M(\lambda, t)=\hat{A}+\hat{V}(t)-\lambda E$. The roots of $\operatorname{det} M(\lambda, t)=0$ are $\lambda_{i}(t)$ $(i=1, \cdots, n)$. Let the cofactor of $m_{i j}(\lambda, t)$ in $M(\lambda, t)$ be $c_{i j}(\lambda, t)$, and let

$$
\hat{s}_{i j}(t)=c_{i j}\left(\lambda_{j}(t), t\right) / \prod_{k \neq j}\left(\sigma_{k}-\sigma_{j}\right) \text {. }
$$

Because $c_{j i}\left(\lambda_{j}(t), t\right)$ tends to the cofactor of the element in the $j$ th row and $i$ th column of $\hat{A}-\sigma_{j} E$ as $t \rightarrow \infty, \lim _{t \rightarrow \infty} \hat{s}_{i j}(t)=\delta_{i j}$. We let $\hat{S}(t)$ be the matrix with elements $\hat{s}_{i j}(t)$, and then it is clear that $\lim _{t \rightarrow \infty} \hat{S}(t)=E$. Also,

$$
\sum_{j=1}^{n}\left(\hat{a}_{k j}+\hat{v}_{k j}(t)-\lambda_{i}(t) \delta_{k j}\right) c_{i j}\left(\lambda_{i}(t), t\right)=0 .
$$


For $\mathbf{i} \neq \mathrm{k}$, this is true because a determinant with two identical rows is zero. For $i=k$, it is true because $\lambda_{i}(t)$ is a characteristic root of $\hat{A}+\hat{V}(t)$. Now (18) and (19) imply that $[\hat{A}+\hat{V}(t)] \hat{S}(t)=\hat{S}(t) \Lambda(t)$. Since $\lim _{t \rightarrow \infty} \hat{S}(t)=E, \hat{S}^{-1}(t)$ exists for large $t$, and

$$
\Lambda=\hat{S}^{-1}(\hat{A}+\hat{V}) \hat{S}=S^{-1}(A+V) S
$$

as desired.

It remains to prove (17). The elements of $\hat{S}^{\prime}(t)$ are linear and homogeneous in the elements of $\hat{V}^{\prime}(t)$ and the $\lambda_{i}^{\prime}(t)$. Because of $(16)$, we need only prove

$$
\int^{\infty} f(t)\left|\lambda_{i}^{\prime}(t)\right| d t<\infty \quad(i=1, \cdots, n) .
$$

Let $F(\lambda, t)=\operatorname{det} M(\lambda, t)$. Since $F\left(\lambda_{i}(t), t\right)=0$,

$$
F_{\lambda}\left(\lambda_{i}(t), t\right) \lambda_{i}^{\prime}(t)+F_{t}\left(\lambda_{i}(t), t\right)=0 .
$$

Because $A$ has distinct characteristic roots, $F_{\lambda}\left(\lambda_{i}(t), t\right)$ tends to a non-zero limit as $t \rightarrow \infty$. The term $F_{t}\left(\lambda_{i}(t), t\right)$ is linear and homogeneous in the elements of $\hat{V}^{\prime}(t)$, and thus (16) implies (20).

By means of Lemma 6, we can compare the solutions of

$$
x^{\prime}=[A+V(t)] x
$$

and

$$
y^{\prime}=[A+V(t)+R(t)] y
$$

We let

$$
\int_{0}^{t} \Re \lambda_{i}(s) d s=\gamma_{i}(t) \quad(i=1, \cdots, n) ; \quad \rho(t)=\gamma_{n}(t)-\gamma_{1}(t)
$$

Our assumptions imply that $\gamma_{1}(\mathrm{t}) \leq \gamma_{2}(\mathrm{t}) \leq \cdots \leq \gamma_{\mathrm{n}}(\mathrm{t})$ for $0 \leq \mathrm{t}<\infty$.

THEOREM 7. Suppose that

$$
\int^{\infty} e^{\rho(t)+k(t)}\left|V^{\prime}(t)\right| d t<\infty, \quad \int^{\infty} e^{\rho(t)+k(t)}|R(t)| d t<\infty,
$$

for some function $\mathrm{k}(\mathrm{t}) \geq 0$. Then, to each solution $\mathrm{x}(\mathrm{t})$ of $(21)$, there corresponds a solution $\mathrm{y}(\mathrm{t})$ of $(22)$ such that $\mathrm{y}(\mathrm{t})-\mathrm{x}(\mathrm{t})=\mathrm{o}\left(\mathrm{e}^{\gamma_{1}(\mathrm{t})-\mathrm{k}(\mathrm{t})}\right)$ as $\mathrm{t} \rightarrow \infty$.

Proof. By Lemma 6, there exists a matrix $\mathrm{S}(\mathrm{t})$ which tends to a non-singular constant matrix $T$ as $t \rightarrow \infty$, and such that $S^{-1}[A+V] S=\Lambda$. The matrices $S(t)$ and $S^{-1}(t)$ are bounded for large $t$, and

$$
\int^{\infty} e^{\rho(t)+k(t)}\left|S^{\prime}(t)\right| d t<\infty .
$$

The changes of variable $\mathrm{x}=\mathrm{S}(\mathrm{t}) \mathrm{w}, \mathrm{y}=\mathrm{S}(\mathrm{t}) \mathrm{z}$ transform (21) to 


$$
w^{\prime}=\left[\Lambda(t)+B_{0}(t)\right] w, \quad B_{0}(t)=-S^{-1}(t) S^{\prime}(t),
$$

and (22) to

$$
z^{\prime}=\left[\Lambda(t)+B_{0}(t)+B(t)\right] z, \quad B(t)=S^{-1}(t) R(t) S(t) .
$$

Because of (23) and (24),

$$
\int^{\infty} \mathrm{e}^{\rho(\mathrm{t})+\mathrm{k}(\mathrm{t})}|\mathrm{B}(\mathrm{t})| d t<\infty, \quad \int^{\infty} \mathrm{e}^{\rho(\mathrm{t})+\mathrm{k}(\mathrm{t})}\left|\mathrm{B}_{0}(\mathrm{t})\right| d t<\infty .
$$

We compare (25) and (26) with

$$
u^{\prime}=\Lambda(t) u,
$$

which has a fundamental matrix $U(t)$ such that

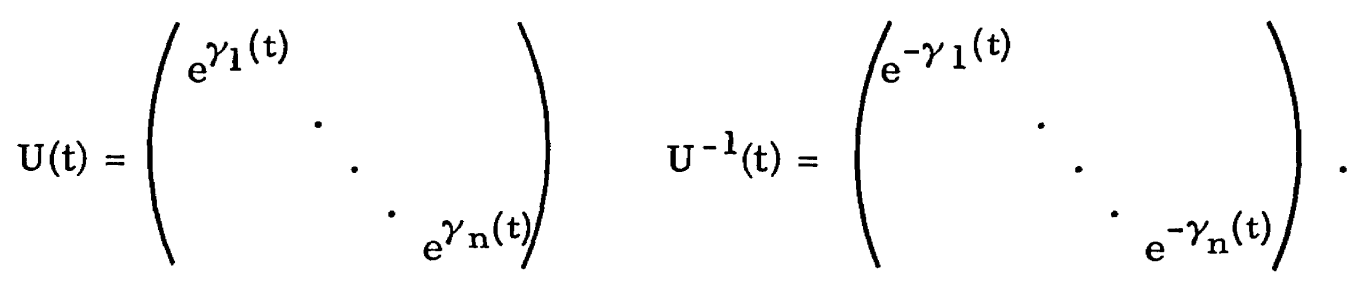

In comparing (25) with (28) as in Theorem 4, we are led to consider the system $v^{\prime}=U^{-1}(t) B_{0}(t) U(t) v$, or

$$
v_{i}^{\prime}=\sum_{j=1}^{n} e^{-\gamma_{i}(t)} B_{0 i j}(t) e^{\gamma_{j}(t)} v_{j},
$$

where $v_{i}$ is the $i$ th component of $v$. The maximum coefficient in (29) is

$$
e^{\gamma_{n}(t)-\gamma_{i}(t)}\left|B_{0 i n}(t)\right|=e^{\gamma_{1}(t)-\gamma_{i}(t)-k(t)} q_{i}(t),
$$

where $q_{i}(t)$ is a non-negative integrable function because of (27). We follow the argument of Theorem 4, noting that $\mathrm{e}^{\gamma_{1}(t)-\gamma_{i}(t)-k(t)}$ is monotone decreasing since $\gamma_{1}(t)-\gamma_{i}(t)-k(t) \leq \gamma_{1}(t)-\gamma_{i}(t) \leq 0$. We see that there exists a correspondence between components $v_{i}(t)$ of solutions of (29) and components $c_{i}$ of any constant vector $\mathrm{c}$ such that

$$
\left|v_{i}(t)-c_{i}\right|=o\left(e^{\gamma_{1}(t)-\gamma_{i}(t)-k(t)}\right) \quad(t \rightarrow \infty)
$$

This leads to a correspondence between solutions $u(t)=U(t) c$ of $(28)$ and $\mathrm{w}(\mathrm{t})=\mathrm{U}(\mathrm{t}) \mathrm{v}(\mathrm{t})$ of $(25)$ such that

$$
|u(t)-w(t)|=o\left(e^{\gamma_{1}(t)-k(t)}\right) \quad(t \rightarrow \infty) .
$$

An analogous argument yields a correspondence between solutions $u(t)$ of (28) and $z(t)$ of $(26)$ such that

$$
|u(t)-z(t)|=o\left(e^{\gamma_{1}(t)-k(t)}\right) \quad(t \rightarrow \infty)
$$


Consequently,

$$
|z(t)-w(t)|=o\left(e^{\gamma_{1}(t)-k(t)}\right) \quad(t \rightarrow \infty)
$$

Since $S(t)$ and $S^{-1}(t)$ are bounded for large $t$, this implies the conclusion of the theorem.

If $\gamma_{1}(t) \geq 0$, we can take $k(t)=\gamma_{1}(t)$, and we see that

$$
\int^{\infty} e^{\gamma_{n}(t)}\left|V^{\prime}(t)\right| d t<\infty, \quad \int^{\infty} e^{\gamma_{n}(t)}|R(t)| d t<\infty
$$

implies $\mathrm{y}(\mathrm{t})-\mathrm{x}(\mathrm{t})=\mathrm{o}(1)$.

The standard results of this type ([2], [3, p. 38], [4, p. 92]) differ from Theorem 7 in that they require only integrability of $\left|V^{\prime}(t)\right|$ and $|R(t)|$, and give somewhat different error estimates. They are sharper in the sense that they can single out a particular solution of (21) for estimates, while we must always consider the worst possible solution.

\section{REFERENCES}

1. F. Brauer, Global behaviour of solutions of ordinary differential equations, J. Math. Analysis Appl. 2 (1961), 145-158.

2. L. Cesari, Un nuovo criterio di stabilità per le soluzioni delle equazioni differenziali lineari, Ann. Scuola Norm. Super. Pisa (2) 9 (1940), 163-186.

3. - Asymptotic behavior and stability problems in ordinary differential equations, Ergebnisse Math. Grenzgebiete N.F. 16, Springer-Verlag, Berlin (1959).

4. E. A. Coddington and N. Levinson, Theory of ordinary differential equations, McGraw-Hill, New York (1955).

5. R. Conti, Sulla prolungabilità delle soluzioni di un sistema di equazioni differenziali ordinarie, Boll. Un. Mat. Ital. (3) 11 (1956), 510-514.

6. N. Levinson, The asymptotic nature of solutions of linear systems of differential equations, Duke. Math. J. 15 (1948), 111-126.

7. A. Wintner, Small perturbations, Amer. J. Math. 67 (1945), 417-430.

8. - Linear variation of constants, Amer. J. Math. 68 (1946), 185-213.

9. - , An abelian lemma concerning asymptotic equilibria, Amer. J. Math. 68 (1946), 451-454.

10. - Asymptotic integration constants, Amer. J. Math. 68 (1946), 553-559.

11. - Ordinary differential equations and Laplace transforms, Amer. J. Math. 79 (1957), 265-294.

12. V. A. Yakubovič, On the asymptotic behavior of the solutions of a system of differential equations (Russian), Mat. Sbornik (N.S.) 28 (70) (1951), 217-240. 
